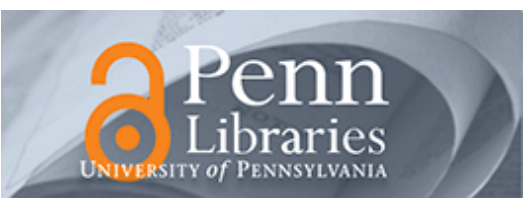

University of Pennsylvania ScholarlyCommons

February 2007

\title{
Pharmacokinetic and behavioral characterization of a longterm antipsychotic delivery system in rodents and rabbits
}

\author{
Kayla L. Metzger \\ University of Pennsylvania \\ Jody M. Shoemaker \\ University of California \\ Jonathan B. Kahn \\ University of Pennsylvania \\ Christina R. Maxwell \\ University of Pennsylvania \\ Yuling Liang \\ University of Pennsylvania
}

See next page for additional authors

Follow this and additional works at: https://repository.upenn.edu/mse_papers

\section{Recommended Citation}

Metzger, K. L., Shoemaker, J. M., Kahn, J. B., Maxwell, C. R., Liang, Y., Tokarczyk, J., Kanes, S. J., Hans, M., Lowman, A. M., Dan, N., Winey, K. I., Swerdlow, N. R., \& Siegel, S. J. (2007). Pharmacokinetic and behavioral characterization of a longterm antipsychotic delivery system in rodents and rabbits. Retrieved from https://repository.upenn.edu/mse_papers/122

Postprint version. Published in Psychopharmacology, Volume 190, Issue 2, February 2007, pages 201-211.

Publisher URL: http://dx.doi.org/10.1007/s00213-006-0616-8

This paper is posted at ScholarlyCommons. https://repository.upenn.edu/mse_papers/122

For more information, please contact repository@pobox.upenn.edu. 


\title{
Pharmacokinetic and behavioral characterization of a longterm antipsychotic delivery system in rodents and rabbits
}

\author{
Abstract \\ Rationale: Non-adherence with medication remains the major correctable cause of poor outcome in \\ schizophrenia. However, few treatments have addressed this major determinant of outcome with novel \\ long-term delivery systems. \\ Objectives: The aim of this study was to provide biological proof of concept for a long-term implantable \\ antipsychotic delivery system in rodents and rabbits. \\ Materials and methods: Implantable formulations of haloperidol were created using biodegradable \\ polymers. Implants were characterized for in vitro release and in vivo behavior using prepulse inhibition of \\ startle in rats and mice, as well as pharmacokinetics in rabbits. \\ Results: Behavioral measures demonstrate the effectiveness of haloperidol implants delivering $1 \mathrm{mg} / \mathrm{kg}$ \\ in mice and $0.6 \mathrm{mg} / \mathrm{kg}$ in rats to block amphetamine $(10 \mathrm{mg} / \mathrm{kg})$ in mice or apomorphine $(0.5 \mathrm{mg} / \mathrm{kg})$ in \\ rats. Additionally, we demonstrate the pattern of release from single polymer implants for 1 year in \\ rabbits.
}

Conclusions: The current study suggests that implantable formulations are a viable approach to providing long-term delivery of antipsychotic medications in vivo using animal models of behavior and pharmacokinetics. In contrast to depot formulations, implantable formulations could last 6 months or longer. Additionally, implants can be removed throughout the delivery interval, offering a degree of reversibility not available with depot formulations.

\section{Keywords}

antipsychotic agents, treatment adherence, schizophrenia, drug delivery systems, drug implants, prepulse inhibition, haloperidol, rat, mouse, rabbit

\section{Comments}

Postprint version. Published in Psychopharmacology, Volume 190, Issue 2, February 2007, pages 201-211.

Publisher URL: http://dx.doi.org/10.1007/s00213-006-0616-8

\section{Author(s)}

Kayla L. Metzger, Jody M. Shoemaker, Jonathan B. Kahn, Christina R. Maxwell, Yuling Liang, Jan Tokarczyk, Stephen J. Kanes, Meredith Hans, Anthony M. Lowman, Nily Dan, Karen I. Winey, Neal R. Swerdlow, and Steven J. Siegel 


\title{
Pharmacokinetic and behavioral characterization of a long- term antipsychotic delivery system in rodents and rabbits
}

\author{
Kayla L. Metzger • Jody M. Shoemaker • \\ Jonathan B. Kahn • Christina R. Maxwell • \\ Yuling Liang • Jan Tokarczyk • Stephen J. Kanes • \\ Meredith Hans • Anthony M. Lowman • Nily Dan • \\ Karen I. Winey • Neal R. Swerdlow • Steven J. Siegel
}

Received: 31 May 2006 / Accepted: 13 October 2006

(C) Springer-Verlag 2006

\begin{abstract}
Rationale Non-adherence with medication remains the major correctable cause of poor outcome in schizophrenia. However, few treatments have addressed this major determinant of outcome with novel long-term delivery systems.

Objectives The aim of this study was to provide biological proof of concept for a long-term implantable antipsychotic delivery system in rodents and rabbits.

Materials and methods Implantable formulations of haloperidol were created using biodegradable polymers. Implants were characterized for in vitro release and in vivo
\end{abstract}

K. L. Metzger · J. B. Kahn · C. R. Maxwell · Y. Liang •

S. J. Siegel

Stanley Center for Experimental Therapeutics in Psychiatry,

Translational Research Laboratories, University of Pennsylvania, Room 2223, 125 South 31st Street,

Philadelphia, PA 19104, USA

K. L. Metzger · J. B. Kahn • C. R. Maxwell · Y. Liang •

J. Tokarczyk $\cdot$ S. J. Kanes $\cdot$ S. J. Siegel $(\bowtie)$

Division of Neuropsychiatry, Department of Psychiatry,

University of Pennsylvania,

Philadelphia, PA, USA

e-mail: siegels@mail.med.upenn.edu

J. M. Shoemaker $\cdot$ N. R. Swerdlow

Department of Psychiatry, University of California,

San Diego, CA, USA

M. Hans $\cdot$ A. M. Lowman $\cdot$ N. Dan

Department of Chemical Engineering, Drexel University,

Philadelphia, PA, USA

K. I. Winey

Department of Materials Science and Engineering,

University of Pennsylvania,

Philadelphia, PA, USA behavior using prepulse inhibition of startle in rats and mice, as well as pharmacokinetics in rabbits.

Results Behavioral measures demonstrate the effectiveness of haloperidol implants delivering $1 \mathrm{mg} / \mathrm{kg}$ in mice and $0.6 \mathrm{mg} / \mathrm{kg}$ in rats to block amphetamine $(10 \mathrm{mg} / \mathrm{kg})$ in mice or apomorphine $(0.5 \mathrm{mg} / \mathrm{kg})$ in rats. Additionally, we demonstrate the pattern of release from single polymer implants for 1 year in rabbits.

Conclusions The current study suggests that implantable formulations are a viable approach to providing long-term delivery of antipsychotic medications in vivo using animal models of behavior and pharmacokinetics. In contrast to depot formulations, implantable formulations could last 6 months or longer. Additionally, implants can be removed throughout the delivery interval, offering a degree of reversibility not available with depot formulations.

Keywords Antipsychotic agents - Treatment adherence . Schizophrenia $\cdot$ Drug delivery systems · Drug implants . Prepulse inhibition $\cdot$ Haloperidol $\cdot$ Rat $\cdot$ Mouse $\cdot$ Rabbit

\section{Introduction}

Non-adherence with prescribed therapeutics remains the highest predictor of patient relapse and rehospitalization (Ayuso-Gutierrez and del Rio Vega 1997; Harris et al. 2002; Lieberman et al. 2005; Olfson et al. 2000; Svarstad et al. 2001). Up to $55 \%$ of individuals with schizophrenia have significant difficulties adhering to treatment (Corriss et al. 1999). Non-adherent patients are twice as likely to undergo rehospitalization from relapse (Svarstad et al. 2001), resulting in a poor quality of life. A Medicaid study found that only $11.6 \%$ of treated patients achieved 1 year of 
uninterrupted antipsychotic medication therapy (McCombs et al. 1999). Economically, the social burden of healthcare costs for non-adherence in mental illnesses like schizophrenia has been estimated at $\$ 2.3$ billion annually (Menzin et al. 2003). Another study found an actual medicine adherence rate of $23 \%$ to be in stark contrast with a patient-reported rate of $55 \%$, a larger than twofold difference (Velligan et al. 2003), suggesting that nonadherence is a greater problem than previously thought.

There are multiple causes of non-adherence in schizophrenia ranging from adverse drug side effects to lack of family support (Robinson et al. 2002), but previous aims at improving adherence through parenteral administrations of antipsychotic agents have proven effective. Depot injections, in particular, have worked well to deliver antipsychotic medications over long periods of time with enhanced efficacy over oral dosing (Adams et al. 2001; Gastpar et al. 2005). However, limitations of depot formulations restrict more significant improvements in adherence and efficacy. For example, adverse side effects are occasionally manifested and must be endured for the remainder of the treatment interval due to the inability to remove depot medications. Prolonged pain often persists at repeated injection sites and as a result, many patients cease to return for continued clinical treatment (Kane et al. 1998). Decanoate formulations are further limited by chemistry, with many compounds unable to form the required ester linkages. Although studies demonstrate promising results with risperidone depots over the course of 1 year of treatment (Martin et al. 2003), injections must be administered bimonthly, requiring 24 annual treatment decisions by patients, and therefore may not provide adequate sustained care to many patients with schizophrenia.

Accordingly, we have investigated a new method of long-term implantable medication delivery to improve adherence in the treatment of schizophrenia. Implantable systems have the capability to optimize a medication's therapeutic properties, rendering treatments that are more safe, efficacious, and reliable (Dash and Cudworth 1998). The gastrointestinal tract is bypassed; therefore, less medication is generally required, and side effects can be minimized. Implantable systems can also be easily removed in case of adverse side effects, offering a degree of reversibility not available with depot injections. With biodegradable systems advancing further in the field of drug delivery, required removal surgery is eliminated, cutting in half the inherent invasiveness to the patient (Fischel-Ghodsian and Newton 1993).

Many biodegradable implant systems rely on a polylactide-co-glycolide (PLGA) polymer which is highly biocompatible and physically strong (Kitchell and Wise 1985). Its degradation products, lactic acid and glycolic acid, are water soluble, non-toxic products of normal metabolism that are either excreted or further metabolized to carbon dioxide and water in the Krebs (citric acid) cycle (Curtis 1983; Okada and Toguchi 1995). A limited number of systems already utilize PLGA and other biocompatible polymers to successfully achieve long-term delivery, including devices to control thyrotropin-releasing hormone in controlling metabolism (Okada and Toguchi 1995), L-dopa to treat Parkinson's disease (Sabel et al. 1990), and naltrexone to treat narcotic addiction (Sharon and Wise 1981). Several intraocular systems, including Vitrasert ${ }^{\circledR}$ (Bausch and Lomb), offer biocompatible delivery systems with controlled-release drug therapy for periods ranging from several days to 1 year.

The current report provides proof of concept for this new method of drug delivery in psychiatry rather than an introduction of a specific product. Additionally, this research is sponsored by the Stanley Medical Research Institute, had no support from industry, and thus, has no inherent limitations regarding future application to a broad array of compounds. Therefore, we have applied this technology to the high potency agent haloperidol to minimize the size of implant needed for effectiveness. The current study examines in vitro and in vivo kinetics as well as behavioral testing to determine the viability of the implantable system.

\section{Materials and methods}

In vitro release studies

Implants were fabricated from a PLGA Medisorb ${ }^{\circledR}$ bioabsorbable polymer (Alkermes, Cincinnati, $\mathrm{OH}$ ) and haloperidol (Sigma, St. Louis, MO). The polymer was composed of PLGA in a molar ratio of 50:50. Polymer and drug were mixed in a ratio of $80: 20$ by mass and solvent cast. The preparation was evaporated at $40^{\circ} \mathrm{C}$ in a vacuum oven under light airflow until no solvent remained and the mixture had returned to its constituent mass. The resulting film was compression molded under a force of $25,000 \mathrm{lbs}$ at $80^{\circ} \mathrm{C}$ for $3 \mathrm{~min}$. Negative control implants with $0 \%$ drug load were fabricated in the same manner.

Each implant was added to a separate amber-glass dissolution jar (Wheaton, Millville, NJ) containing $500 \mathrm{ml}$ phosphate-buffered saline solution at $\mathrm{pH}$ 7.4. The jars were stored in the dark at $37.4^{\circ} \mathrm{C}$ on a shaker at $40 \mathrm{rpm}$ to mimic biological conditions. Aliquots of $1 \mathrm{ml}$ were taken from each jar three times per week and analyzed by ultraviolet spectrophotometry (Hitachi, Los Angeles, CA). Standard curves were prepared for each drug in phosphate-buffered saline. Peak absorbency of $249 \mathrm{~nm}$ was determined before analysis. Assays included negative controls at every time point and a $20-\mu \mathrm{g} / \mathrm{ml}$ positive control of drug in phosphatebuffered saline to assess stability over time. 
In vivo studies

The Institutional Animal Care and Uses Committee (IACUC) at the University of Pennsylvania or University of California at San Diego approved all protocols, and animals were housed in Association for Assessment and Accreditation of Laboratory Animal Care (AAALAC)accredited animal facilities.

Behavioral studies in mice (conducted at University of Pennsylvania) The behavioral effects of haloperidol implants were tested using prepulse inhibition (PPI) of startle in 32 C57BL/6J mice (Jackson Laboratory, Bar Harbor, ME). Implants were fabricated as described above and were designed to deliver $1 \mathrm{mg} / \mathrm{kg} /$ day (Siegel et al. 2002). PLGA-haloperidol implants fabricated with this method have been shown to yield serum concentration of $8.2 \mathrm{nmol} / 1$ (3.1 ng/ml) in mice (Talbot et al. 2004). This concentration is comparable to the haloperidol concentration in humans that yields $75 \% \mathrm{D}_{2}$ receptor occupancy (7.3 nmol/1, $2.75 \mathrm{ng} / \mathrm{ml}$ ), suggesting that the behavioral effects of implants were tested at clinically relevant receptor occupancy (Regenthal et al. 1997).

Implants were placed in the subcutaneous space on the dorsal surface under isoflurane anesthesia (Fig. 1). Animals received either haloperidol $(n=16)$ or blank implants $(n=16)$. Mice were then tested at 21 days after implantation using previously described protocols for PPI (Gould et al. 2004). Each animal was tested after an injection of either vehicle ( $n=16$ ) or D-amphetamine (Sigma) $10 \mathrm{mg} / \mathrm{kg}$ i.p. $(n=16)$, resulting in eight animals in each of the four conditions. Analyses were performed using a between-groups design. Each trial started with a 5-min acclimation period with a 65-dB acoustic background noise followed by a train of five $120-\mathrm{dB}$ startle pulses in an effort to make subsequent startle trials less variable. Startle trials consisted of 40-ms pulses at 0 (no stimulus), 90, 95, 100, 105, 110, 115, and $120 \mathrm{~dB}$. Each stimulus was presented five times in a randomized order with an intertrial interval randomized from 10-20 s. Startle trials were followed by PPI trials.
Each prepulse was $20 \mathrm{~ms}$ in duration followed by a 40-ms startle stimulus of $120 \mathrm{~dB}$. There was a 100 -ms interstimulus interval between prepulse and startle stimuli presentation. PPI was recorded for prepulse intensities of 69,73 , and $81 \mathrm{~dB}$ and no stimulus. Each prepulse trial was administered five times in a random order. Data were collected as 60 1-ms voltage readings, which were averaged over the collection interval to give an average measure for each trial. The entire session in mice lasted $25 \mathrm{~min}$.

Behavioral studies in rats (conducted at University of California, San Diego) A total of 52 rats were utilized in this study. Implants were designed to release $0.6 \mathrm{mg} \mathrm{kg}^{-1}$ day $^{-1}$ of haloperidol (Sigma). Subcutaneous implantation of drug $(n=31)$ or control $(n=21)$ implants was performed under isoflurane anesthesia. Serum haloperidol levels were determined using 20 adult male rats with haloperidol implants on days $14(n=10), 21(n=5)$, and $28(n=5)$ and an additional ten adult male rats with control implants on days $14(n=4), 21(n=3)$, and $28(n=3)$. The behavioral effects of haloperidol implants were tested using PPI in an additional 22 adult male Sprague Dawley rats (Harlan Laboratories, San Diego), weighing 250-350 g, using previously described methods for this species (Swerdlow and Geyer 1998). Seven or 14 days after surgery, rats were exposed to a brief "matching" startle session. Rats were placed in a startle chamber (SR-LAB; San Diego Instruments, San Diego, CA) and exposed to $5 \mathrm{~min}$ of $70-\mathrm{dB}$ background noise followed by 17 pulse trials of $40 \mathrm{~ms} 120-$ $\mathrm{dB}$ noise bursts (pulse) and three prepulse + pulse trials consisting of a $20 \mathrm{~ms} 82-\mathrm{dB}$ (12 dB above background) prepulse followed $100 \mathrm{~ms}$ by a $120-\mathrm{dB}$ pulse (onset to onset). Data from this session were used to assign rats to balanced dose groups according to their average \%PPI.

Behavioral testing continued 2 days after the matching session. Apomorphine (APO; Sigma) at a dose of $0.5 \mathrm{mg} / \mathrm{kg}$ s.c. or $0.1 \%$ ascorbate/saline vehicle was administered to rats immediately before testing. The test session lasted 19 min and was used to assess the effects of haloperidol implants on the PPI-disruptive effects of APO. The session

Fig. 1 Insertion of a rod-shaped PLGA implant into the subcutaneous dorsal space of the mouse. a The implant is placed in the subcutaneous space of a mouse through a 4-mm hole. A $1-\mathrm{cm}$ rod before implantation is displayed in the insert. b An implant is shown in situ after closing with a single stitch. c The mouse shows no signs of distress in its home cage 10 min after implantation

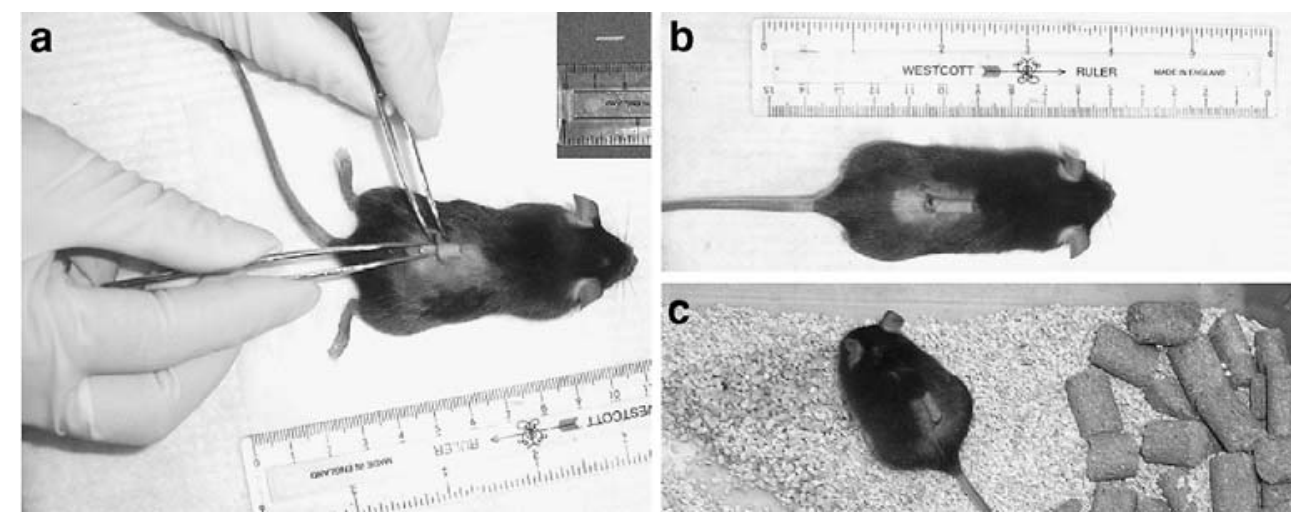


consisted of 5 min of 70-dB background followed by five trial types: pulse noise bursts, prepulse + pulse trials $(20-\mathrm{ms}$ noise bursts 5,10 , or $15 \mathrm{~dB}$ above background followed $100 \mathrm{~ms}$ by a pulse) and no stimulus (nostim) trials. The session consisted of initial and final blocks of four pulse trials separated by two blocks that included eight pulse trials and 15 prepulse + pulse trials (the latter divided equally among 5, 10, and 15-dB prepulse intensities); nostim trials were interspersed between all trials. For these "nostim" trials, stabilimeter readings were recorded during periods where no stimulus was presented; these trials were used to assess gross motor activity during the test session but were not included in the calculation of intertrial intervals, which were variable and averaged $15 \mathrm{~s}$. Four days later, testing was repeated, with each rat receiving either vehicle or APO. To understand the effectiveness of the implants over time, one group of rats was "matched" 1 week post-surgery, and the other group was matched at 2 weeks. All subsequent testing was completed in the same timeline.

For comparison to sustained release implants, the PPIdisruptive effects of APO were also assessed in separate rats treated with acute haloperidol. APO $(0.5 \mathrm{mg} / \mathrm{kg} \mathrm{s.c.})$ or $0.1 \%$ ascorbate/saline vehicle was administered to 16 rats $10 \mathrm{~min}$ after injection of haloperidol (Novaplus, Bedford Laboratories, Bedford, $\mathrm{OH} ; 0.1 \mathrm{mg} / \mathrm{kg}$ s.c.) or lactic acid/saline vehicle. Testing was otherwise identical to that completed with rats that had received sustained-release implants.

Data analysis PPI was calculated as a percent reduction in startle magnitude on prepulse + pulse trials compared to pulse trials. Raw startle data were also analyzed to verify that changes in \%PPI reflected changes in the startleinhibiting effects of prepulses. Data in rats were analyzed using repeated measures analysis of variance (ANOVA) with implantation/pretreatment and implant interval (9 or 16 days) as between-subject factors, and APO, block, and trial type as within-subject repeated measures. In mice, startle units and \%PPI were analyzed using repeated measures ANOVA for overall effect and one-way ANOVA at each decibel intensity. Significant interactions of $p<0.05$ were further explored using Fisher least significant differences post-hoc analyses.

Pharmacokinetic studies in rabbits Rabbits ( $n=6$, Covance Research Products, Denver, PA) ranged in size from 4.0 to $5.7 \mathrm{~kg}$. Five animals received poly-lactide (PLA)haloperidol implants designed to deliver $1 \mathrm{mg} \mathrm{kg}^{-1}$ day $^{-1}$ for an anticipated delivery period of 1 year. One rabbit received implants of PLA without drug as a negative control. Rabbits were anesthetized with ketamine/xylazine $(35: 5 \mathrm{mg} / \mathrm{kg})$ before s.c. implantation.

Rabbits were vasodilated with acepromazine $(1 \mathrm{mg} / \mathrm{kg})$ twice per month for 12 months to draw $5-\mathrm{ml}$ blood samples from the ear. Whole blood was centrifuged, and haloperidol was extracted from serum with an Oasis MCX solid phase extraction column (Waters, Milford, MA). Analysis was performed by HPLC with UV detection at $254 \mathrm{~nm}$. A standard curve for the assay was made with extracted serum, and the retention time for haloperidol was determined from positive control samples within each run. The positive control condition consisted of haloperidol spiked in serum as well as haloperidol in mobile phase. Negative controls were also assayed at each time point. Rabbits were sacrificed after 365 days and the remains of implants removed.

Constituents of residual implants were determined by ${ }^{1} \mathrm{H}$ NMR. Nuclear magnetic resonance (NMR) was performed at $25^{\circ} \mathrm{C}$ on a Varian Unity Inova $300 \mathrm{MHz}$ instrument, and spectra were analyzed using VNMR 6.1b software (Varian, Palo Alto, CA). Control samples of PLA (Alkermes 100DL High IV) and haloperidol were run in dimethyl sulfoxide (DMSO) and chloroform to define peaks of interest for each compound. Implants were initially dissolved in DMSO- $\mathrm{d}_{6}$, and residual solids were then removed by filtration and dissolved in $\mathrm{CDCl}_{3}$ (chloroform).

\section{Results}

In vitro release studies

The pattern of in vitro drug release from single-polymer implants is shown in Fig. 2a with data expressed as percent total drug content. Full release for 50:50 PLGA implants, similar to those used in behavioral studies in mice, was reached at 40 days. The pattern was characterized by a period of relatively slow release for the first 10 days. This was followed by a steady, more rapid release until day 35 and another slow release period until the implant was depleted. Figure $2 b$ demonstrates the appearance of discshaped haloperidol implants over the delivery interval with the implant swelling as it becomes hydrated and growing smaller as the polymer is degraded and drug is released. Note that the entire drug load is released before total disintegration of the implant, suggesting that reversibility of the procedure is retained throughout the delivery interval.

Behavioral studies

Mouse Haloperidol implants were tested using amphetamineinduced disruption of PPI and startle in mice. There was an overall effect of drug condition on acoustic startle response $\left[F_{(3,25)}=3.61, p=0.027\right]$ and a significant interaction between condition and $\mathrm{dB}$ intensity $\left[F_{(21,175)}=2.86, p<0.001\right]$ (Fig. 3b). Post-hoc analyses showed that D-amphetamine decreased startle in control implant mice at the four highest 

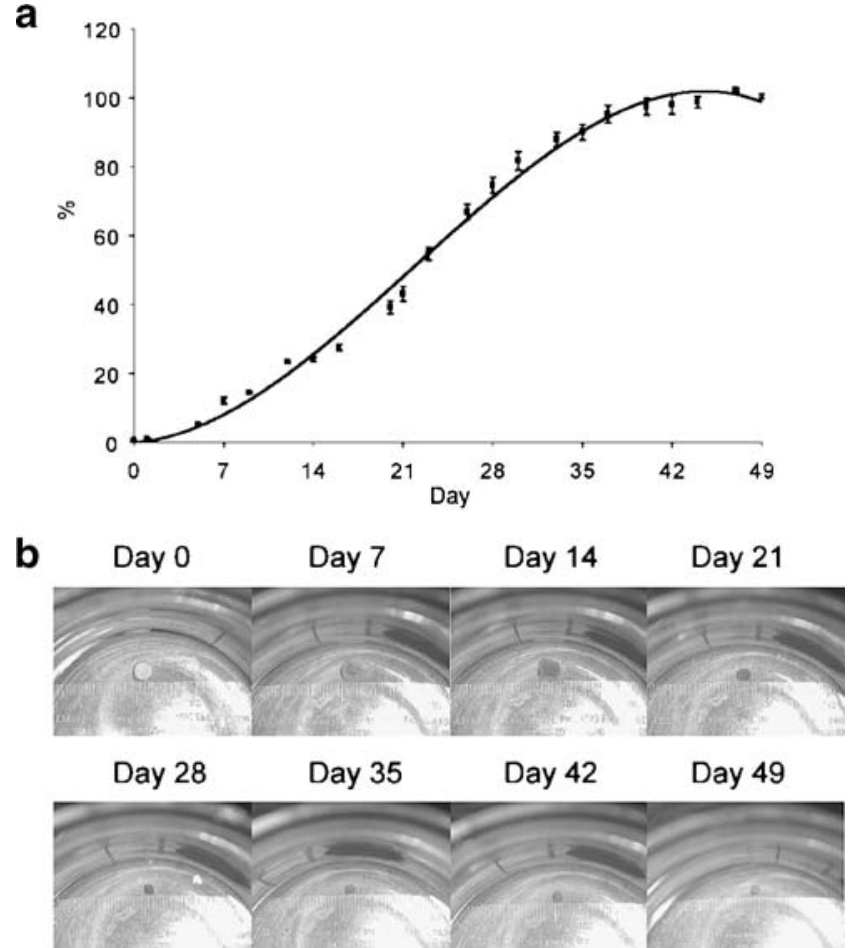

Fig. 2 In vitro release from 20\% haloperidol-loaded pellets of 50:50 4A PLGA polymer. a Data are expressed as percent of total drug released and indicate a slow onset followed by a period of steady rapid release, slowing again to a full release at 40 days. b Photographs of a haloperidol-loaded pellet over 49 days of release show that the implant first swelled as it hydrated and then shrank as drug was released

intensities $(105,110,115$, and $120 \mathrm{~dB})$. This effect was blocked by haloperidol implants at 105, 110, and $120 \mathrm{~dB}$. Additionally, startle response in haloperidol implant mice after amphetamine was not significantly different than control implant mice after saline. Repeated measures ANOVA revealed a marginally significant effect of drug treatments (all implant and injection conditions) for PPI $\left[F_{(3,25)}=2.89\right.$, $p=0.055]$ as well as a significant interaction between treatment conditions and prepulse intensity $\left[F_{(6,50)}=2.41\right.$, $p=0.040$ ] (Fig. 3a). Post-hoc analyses showed that D-amphetamine $(10 \mathrm{mg} / \mathrm{kg})$ reduced PPI at $81 \mathrm{~dB}$ in control animals $(p=0.018)$ and that haloperidol implants reversed this effect (haloperidol implant with amphetamine vs control implant with amphetamine, $p<0.001$ ).

Rat Haloperidol serum levels from implants were $2.3 \pm$ $0.25 \mathrm{ng} / \mathrm{ml}$ at 14 days $(n=10), 1.9 \pm 0.21 \mathrm{ng} / \mathrm{ml}$ at 21 days $(n=5)$, and $0.11 \pm 0.05 \mathrm{ng} / \mathrm{ml}$ at 28 days $(n=5)$. Therefore, PPI and startle experiments in rat were performed before 21 days to ensure appropriate serum concentration. Haloperidol implants significantly attenuated the PPI-disruptive effects of APO (Fig. 4a and b). ANOVA of raw startle magnitude revealed significant main effects of $\mathrm{APO}\left[F_{(1,18)}=\right.$
19.91, $p<0.001]$ and trial type $\left[F_{(3,54)}=38.00, p<0.001\right]$, two-way interactions of APO by trial type $\left[F_{(3,54)}=6.01\right.$, $p=0.001]$ and haloperidol implant by trial type $\left[F_{(3,54)}=\right.$ $7.35, p<0.001]$, and a three-way interaction of implant by APO by trial type $\left[F_{(3,54)}=5.94, p=0.001\right]$. There were no significant effects of post-implant test interval (9 or 16 days) on raw startle magnitude. Inspection of the data (Fig. 4a) reveals the APO-induced loss of the startle-inhibiting effects of prepulses in rats with control implants (left side of figure) and the restoration of these startle-inhibiting effects in rats with haloperidol implants (right side of figure).

ANOVA of PPI revealed significant main effects of APO $\left[F_{(1,18)}=127.04, p<0.001\right]$, haloperidol implant $\left[F_{(1,18)}=33.42, p<0.001\right]$, and prepulse intensity $\left[F_{(2,36)}=\right.$ $43.51, p<0.001]$ along with a significant APO by implant condition interaction $\left[F_{(1,18)}=38.39, p<0.001\right]$. There were no significant effects of post-implant test interval (9 or 16 days) on PPI. Because there was a significant implant condition by APO interaction, pair-wise two-way repeated ANOVA were conducted on the saline and APOtreated groups separately. Post-hoc comparisons revealed a significant effect of haloperidol implant in APO-treated rats $\left[F_{(1,20)}=35.80, p<0.001\right]$, but no effect of haloperidol implant in the saline-treated rats. These effects on PPI reflected a significant APO-induced loss of the startlesuppressing effects of prepulses and an opposition of this effect by haloperidol implants (Fig. 4b). The patterns of haloperidol effects on PPI in implant groups closely parallel the results seen with an acute injection of haloperidol as seen in Fig. 4c. All relevant statistical effects for acute haloperidol were comparable to those reported above for implanted haloperidol as follows. ANOVA revealed significant main effects of haloperidol $\left[F_{(1,14)}=19.58, p<0.001\right]$ and APO $\left[F_{(1,14)}=131.10, p<0.001\right]$ and a significant interaction of haloperidol by APO $\left[F_{(1,14)}=96.48, p<0.001\right]$. Post-hoc analysis confirmed that the basis for this interaction was the significant PPI-enhancing effect of haloperidol in APOtreated rats $\left[F_{(1,14)}=95.47, p<0.001\right]$. There were also significant effects of prepulse intensity $\left[F_{(2,28)}=42.84\right.$, $p<0.001]$ and a significant three-way interaction of prepulse intensity by haloperidol by APO $\left[F_{(2,28)}=5.81, p<0.008\right]$, but no other significant two- three- or four-way interactions.

Pharmacokinetic studies in rabbits

Long-term in vivo pharmacokinetic profile from PLA implants was tested in rabbits to assess the prospect of creating an annual drug delivery system for schizophrenia (Fig. 5). The five rabbits implanted with PLA-haloperidol had detectable serum levels for 360 days with a mean serum concentration of $6.6 \pm 1.1 \mathrm{nmol} / \mathrm{l}(2.5 \pm 0.4 \mathrm{ng} / \mathrm{ml})$. The data are shown with a trendline to illustrate the pattern 
Fig. 3 Behavioral results in implanted mice. Haloperidol implants attenuated amphetamine-induced reduction in startle (a) and PPI (b) in mice. Asterisk indicates $p<0.05$ difference from control implant mice with saline injection. Tau indicates $p<0.05$ difference from control implant mice with amphetamine injection

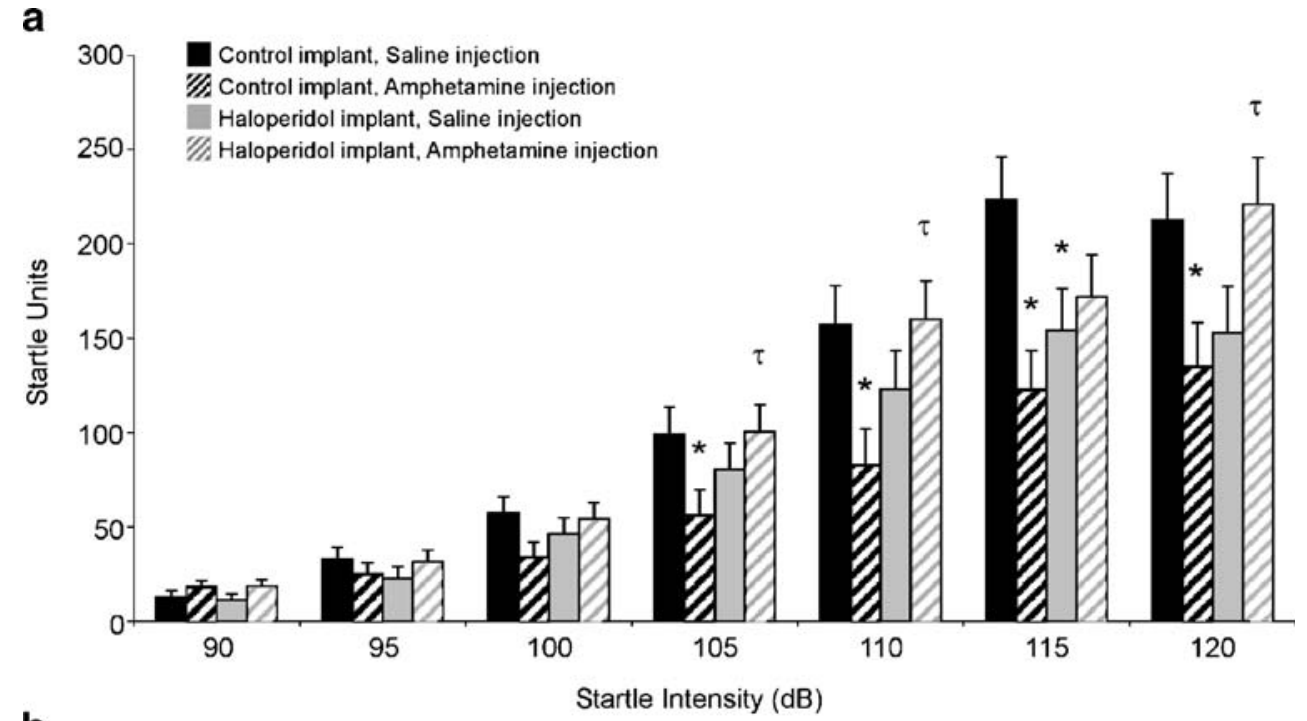

b

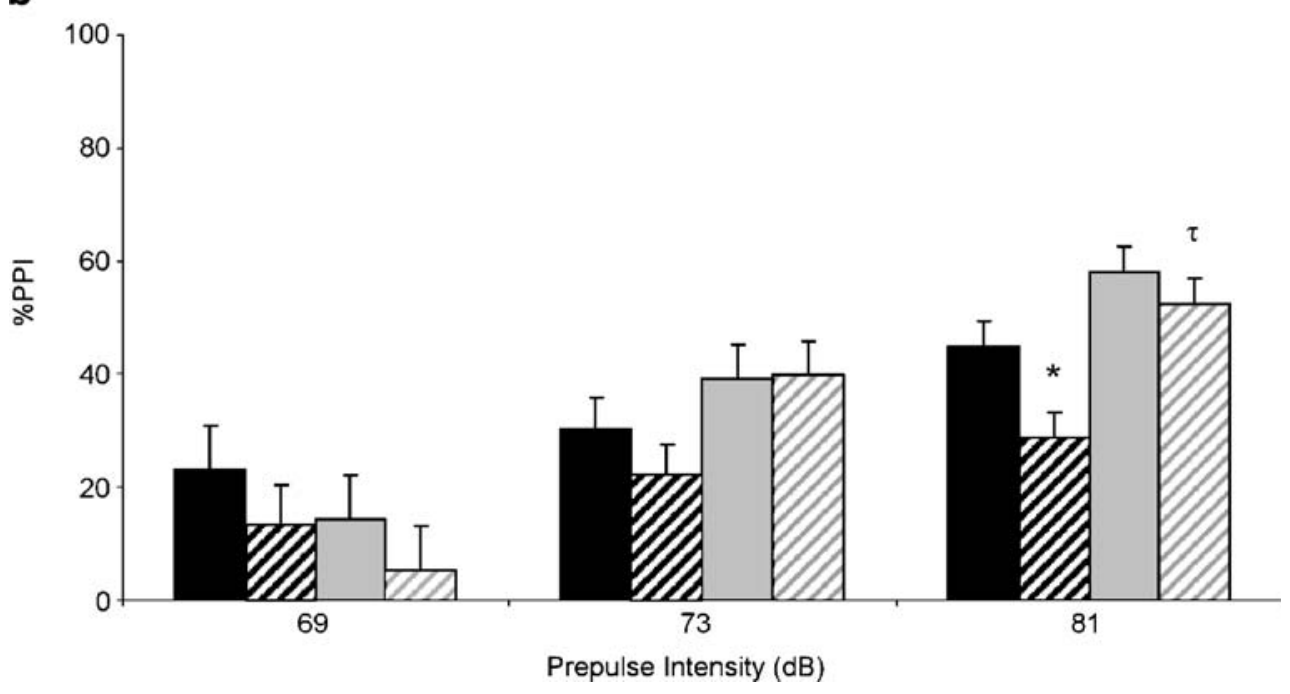

of serum concentration over time. This trendline, which describes the data with a correlation coefficient $\left(R^{2}\right)$ of 0.86, has an approximately symmetrical pattern with maximum values at approximately the $T_{1 / 2}$ for this implant system (6 months). This pattern suggests that a new set of implants could be added every 6 months to oscillate around a desired steady state concentration.

Analyses of residual implant material

No local tissue reaction, fibrosis, or irritation was observed upon implant removal, suggesting good local biocompatibility and reversibility throughout the delivery interval. Residual implants were analyzed using both NMR and HPLC to determine the presence of PLA and haloperidol at the time of removal. HPLC/UV and NMR spectroscopy confirmed the presence of haloperidol and PLGA breakdown products in residual implants. All expected haloperidol peaks were present in DMSO as demonstrated in Fig. 6. Additionally, there were small peaks from 5.2-5.4 ppm that were indicative of the $-\mathrm{CH}$ peak in PLA. However, the corresponding $-\mathrm{CH}_{3}$ peaks from PLA would have been obscured by the haloperidol peaks at low polymer concentrations. The $\mathrm{CDCl}_{3}$ sample contained characteristic haloperidol peaks at 8.1 and $7.4 \mathrm{ppm}$, but did not have the same magnitude of haloperidol peaks as the DMSO sample presumably because most of the haloperidol was extracted in DMSO. The chloroform fraction contained peaks at 0.9 , 1.2, 3.9, and 4.5, consistent with lactic acid, the degradation product of PLA (Fig. 6).

\section{Discussion}

This study demonstrates in vitro and in vivo proof of concept for antipsychotic implants based on biodegradable PLGA copolymers. The results suggest that implantable systems with antipsychotic medications could achieve medication onset within 2 weeks and could be tailored to last up to 6 months according to the needs of the patient. 


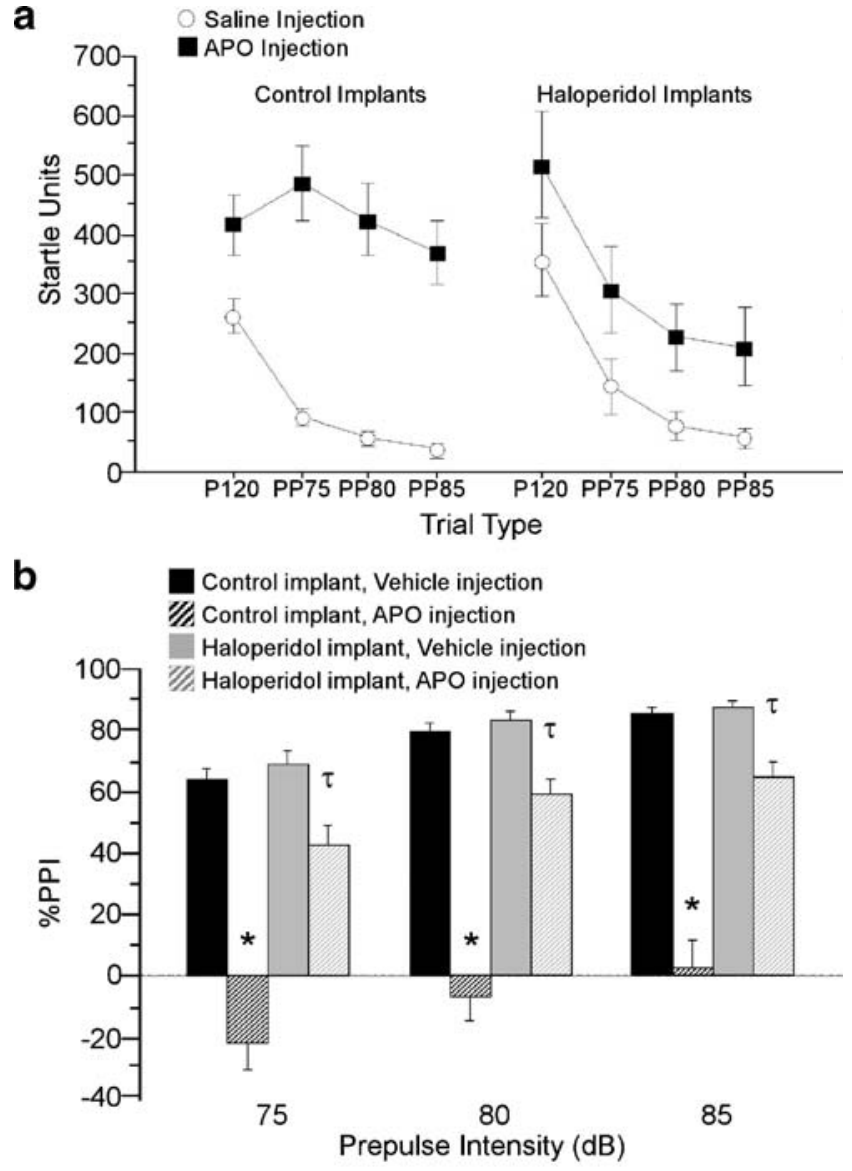

C

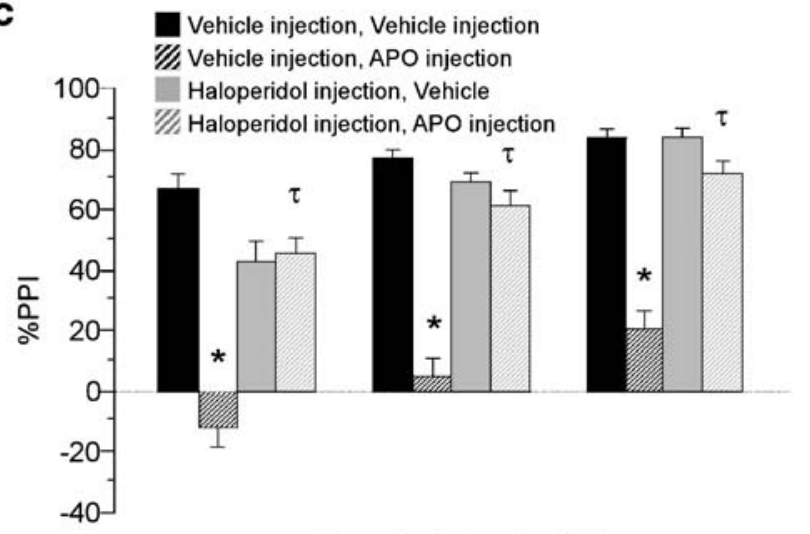

Prepulse Intensity (dB)

Fig. 4 Haloperidol implants attenuated apomorphine-induced reduction of startle (a) and PPI (b) in rats. These effects were comparable to those seen with acute haloperidol injection (c). Asterisk indicates $p<$ 0.05 difference from control implant rats with saline injection. Tau indicates $p<0.05$ difference from control implant rats with apomorphine injection, P120 - startle stimulus alone, PP75-prepulse of $75 \mathrm{~dB}$ followed by startle stimulus, PP85-prepulse of $80 \mathrm{~dB}$ followed by startle stimulus, PP85-prepulse of $85 \mathrm{~dB}$ followed by startle stimulus

Furthermore, the procedure for implantation (and removal, if necessary) could be performed in a short amount of time in an outpatient setting and could be easily tolerated with local anesthetic before subcutaneous insertion. Additional-

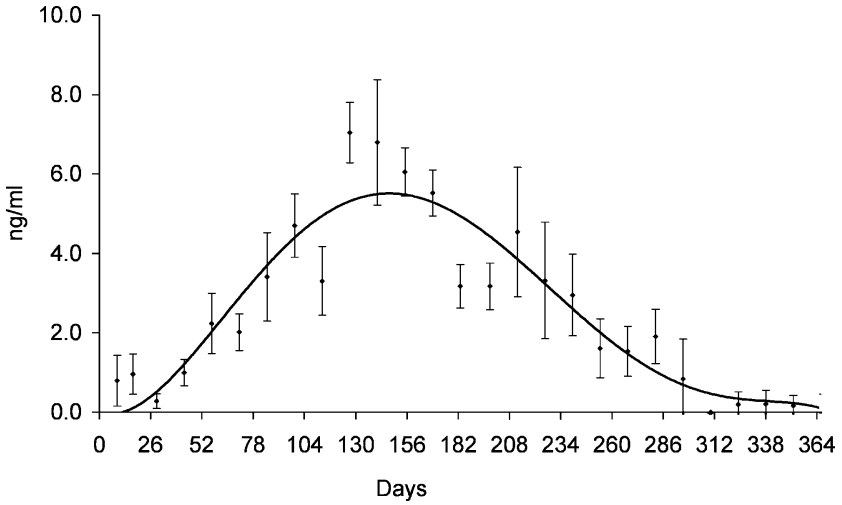

Fig. 5 In vivo haloperidol release from polymer-based implant system in rabbit. Blood samples were evaluated by HPLC for haloperidol concentration. Release is expressed as haloperidol level in nanograms per milliliter. The chart displays data averaged from five animals with a zero-intercept, sixth-degree polynomial average trendline resulting in $R^{2}=0.86$. The negative control resulted in no drug detected (data not shown). The five animals that received PLAhaloperidol implants had a mean haloperidol serum concentration of $2.5 \pm 0.4 \mathrm{ng} / \mathrm{ml}$ over the course of 360 days

ly, many medications that may be limited by chemistry in forming long-lasting decanoates could be incorporated into implantable systems using a variety of polymer matrices.

PPI deficits seen in schizophrenia patients can be modeled using dopamine agonists such as amphetamine and apomorphine. However, acute antipsychotic effects that are often used to reverse these deficits in animal models do not effectively model the steady-state condition achieved in humans, especially due to a short half-life of many drugs in rodents. Mini-pumps are often used to overcome this problem and provide chronic infusion of drug. However, the inability to place relatively large mini-pumps in humans has limited the capacity to have a model that is directly translatable to human use. Alternatively, biodegradable PLGA implants provide a method that can be adapted directly to human use.

In the current study, functional measures of antipsychotic implants were examined using PPI in two species of rodent. Haloperidol implants blocked amphetamine-induced reductions in PPI and startle in mice. Similarly, haloperidol implants blocked apomorphine-induced reductions in PPI in rats, comparable to the effects of acute haloperidol injection. These results indicate that haloperidol implants are effective in this animal model of information processing deficits in schizophrenia, across laboratories, methods, and species.

Long-term pharmacokinetics of haloperidol implants were tested in rabbits over a 1-year period. As evidenced by Fig. 5, the trendline that describes the data for PLAhaloperidol implanted rabbits $\left(R^{2}=0.86\right)$ has a symmetrical pattern with maximum values at approximately 6 months. All negative controls resulted in no drug detected (data not shown). The coherence of polymer implants over time was validated, with the removal of implants occurring at 


\section{a Haloperidol Poly (lactic acid)}

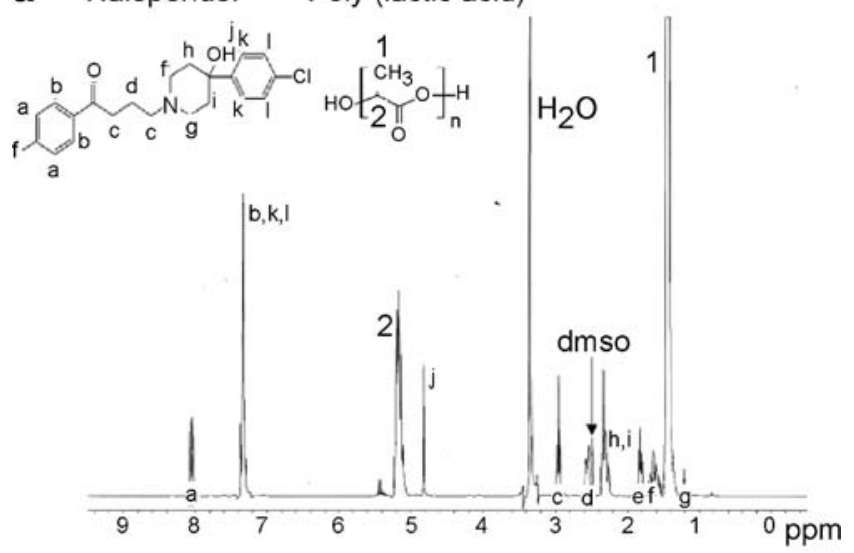

b

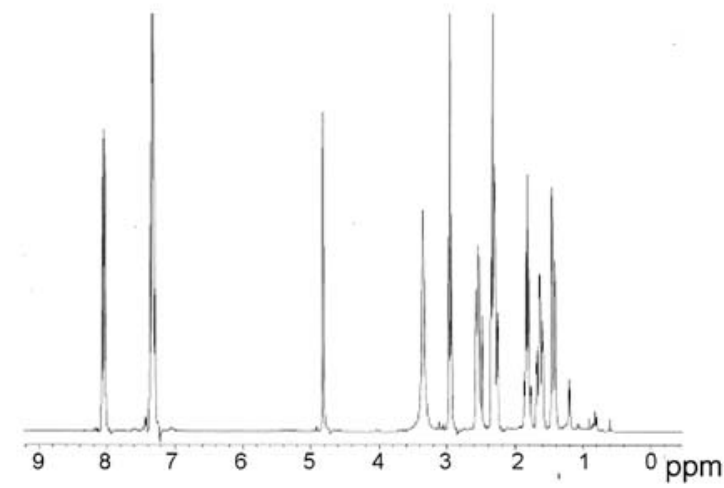

C

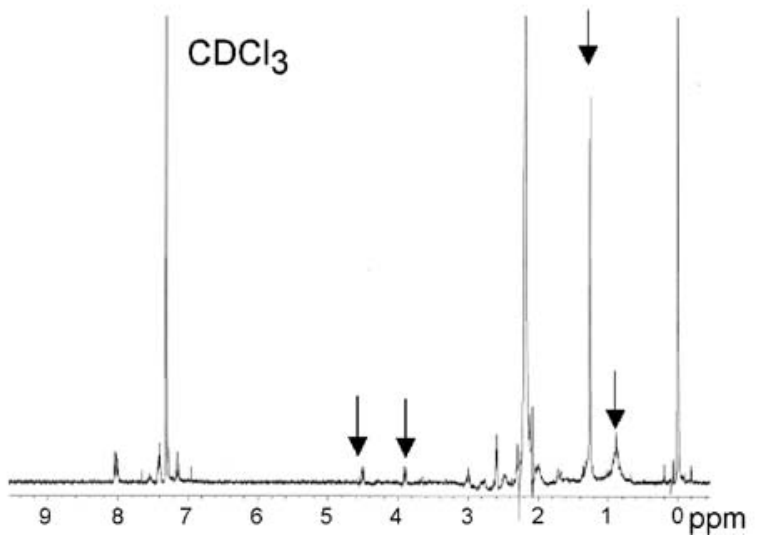

Fig. 6 The top ${ }^{1} \mathrm{H}$ NMR control spectrum shows a PLA and $40 \%$ $(\mathrm{w} / \mathrm{w})$ haloperidol mixture in DMSO- $\mathrm{d}_{6}$. Also depicted are the corresponding chemical structures of haloperidol and PLA. The middle spectrum shows rabbit sample in DMSO- $\mathrm{d}_{6}$ with peaks corresponding to those seen in the control spectrum. The bottom picture shows rabbit sample in chloroform with peaks at 0.9, 1.2, 3.9, and 4.5 consistent with the degradation product of PLA, lactic acid

365 days after implantation. Observation of implants in vitro also showed coherence of the implant after drug was depleted, indicating that removal could be performed if needed well into the duration of implantation. This is a major advantage over existing depot medications using either microspheres (risperidone) or decanoate formulations (haloperidol and fluphenazine).
It is important to note that there are considerable interspecies differences in the metabolism of many drugs, including haloperidol, with rabbits requiring approximately 30-fold higher doses than humans for equivalent plasma concentration. Thus, the absolute doses used in the current study approximate the amount of drug needed for a human despite the difference in body mass. As humans require approximately $50 \mathrm{mg} / \mathrm{month}$ of haloperidol when given as a depot preparation, a 6-month implant system would contain $300 \mathrm{mg}$ of drug. Thus, the implant design used in the current study would necessitate only $750 \mathrm{mg}$ of total material with a $40 \%$ drug load for 1 year of treatment. These materials have a specific gravity of $1.2 \mathrm{~g} / \mathrm{ml}^{2}$, potentially yielding three semi-annual implants with a diameter of $3 \mathrm{~mm}$ and length of $3 \mathrm{~cm}$. Reimplantation every 6 months would result in a gradual escalation to therapeutic level during the first 6 months, followed by small oscillations around a desired serum concentration (Fig. 7a). A series of "starter" polymers with shorter release period could be used at the time of the first implantation to bridge the 6-month lag to therapeutic levels (Fig. 7b).

Although the current formulation utilized haloperidol, other high potency antipsychotic medications including risperidone also show promise for this approach. Whereas many studies have extolled the virtues of such newer agents, recent meta-analyses and controlled clinical trials have questioned the extent to which newer, more costly agents add benefit over low-dose traditional agents (Davis et al. 2003; Geddes et al. 2000; Lieberman et al. 2005; Rosenheck et al. 2003). Since PLGA implants are amenable to many compounds, future studies can incorporate various agents to test their applicability in long-term delivery implants. The stability of each drug in PLGA polymer implants will vary, because as the implant is hydrated, the drug will be exposed to an aqueous environment. Therefore, drugs such as haloperidol and others that are stable in aqueous media for greater than 1 year are preferable for such systems.

There were several limitations to the current study. For example, the selection of animals that were similar in size and that live in a controlled environment limits the ability to fully recreate variances in body mass index, body temperature, drug metabolism, and implant degradation rates that would exist in humans. However, implants were tailored to match animals of approximately $30 \mathrm{~g}$ (mouse), $300 \mathrm{~g}$ (rat) and 5,000 g (rabbit) showing flexibility over two orders of magnitude range of body mass. Drug dosing with implants in a patient population would also need to be tailored to fit the treatment needs of each patient. Once the bioequivalence is established between oral, injectable, and implantable formulations, patients could be dosed based on known relationships and body mass. Both the size and number of implants could be varied based on the indicated 

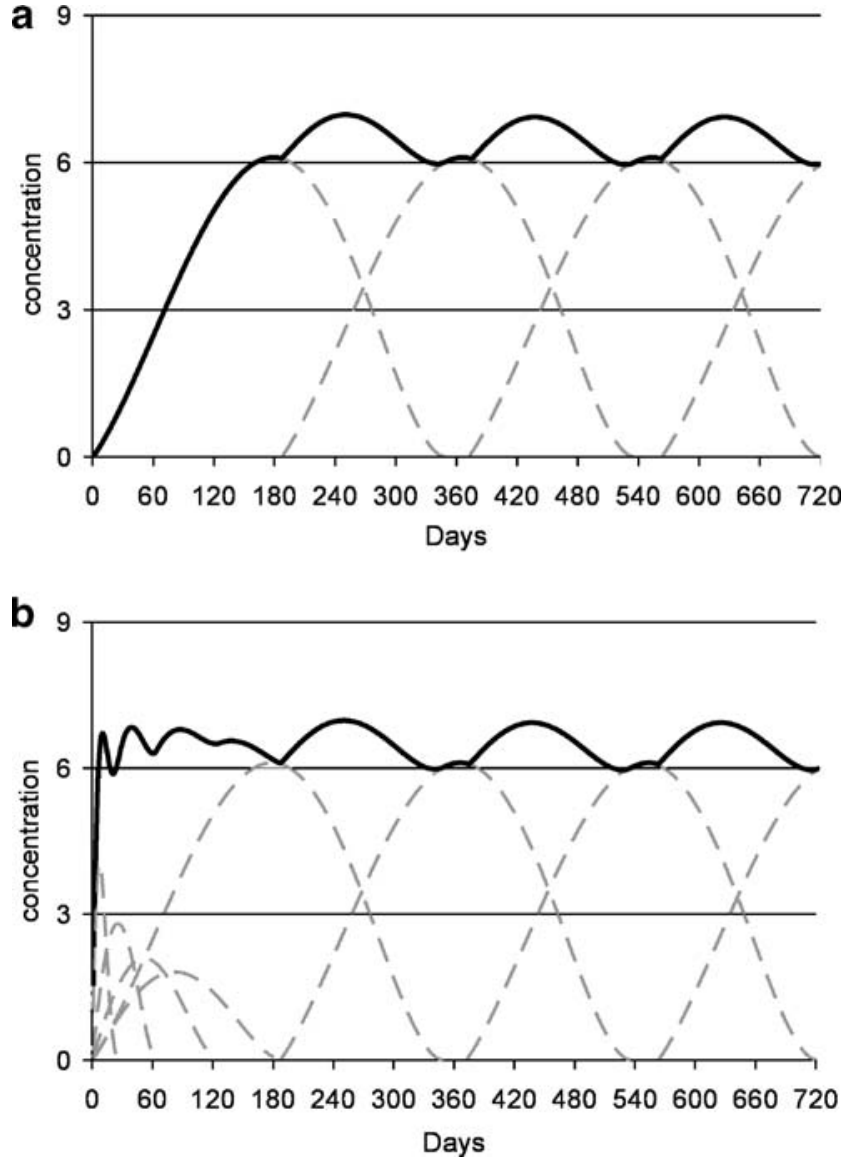

Fig. 7 Representation of ideal multiple polymer-antipsychotic implant system. a Combined release (dark solid line) following reimplantation of a single polymer every 6 months (gray dotted lines). Note that this system would result in a gradual escalation to therapeutic level during the first 6 months followed by small oscillations around a desired serum concentration. b Representation of a five-polymer system in which four rapidly degrading polymers (starter set) are combined with one longer lasting polymer (maintenance set) that is reimplanted every 6 months. Levels that result from all five individual polymers are represented with the solid line, and release profiles from individual polymers are represented by dashed lines. This system could reach target levels in approximately 2 weeks with small oscillations around a target concentration thereafter

dose for that person. For example, modular systems for 0.5 and $1.0 \mathrm{mg} /$ day could be offered to customize implants using these doses alone or in combination (i.e., $1.5 \mathrm{mg}$ /day could be made by combining 1.0 and $0.5 \mathrm{mg}$ /day sets). The current study shows that long-term in vivo release of haloperidol from biodegradable implants is viable for a dosing interval of 2 weeks to 6 months. However, extension of long-term delivery to other medications and medical conditions is desirable. The use of implants to treat psychiatric or nonpsychiatric disorders would necessarily require safeguards to ensure patients' ability to provide informed consent. Although minimally invasive, the procedures for implantation and possible removal still necessitate careful attention to protect patient autonomy. Consistent with this goal, one advantage of surgically implantable systems would be that patients could make decisions regarding long-term treatment during periods of health. After medication treatment, patients are able to achieve significant symptom remission such that they could make decisions to follow long-term medication treatments for the subsequent 6 to 12 months. Previously published and preliminary data from our group suggest that many patients (43\%) and their families (70\%) would support such surgically implantable, long-term methods of treatment if they were available (Irani et al. 2004; Dankert et al., in review).

Whereas many studies report medication adherence rates of $50 \%$, it is increasingly thought that data are skewed, as many patients cease medication treatment once outside clinical trials (Seeman 2001). However, when the physician and patient come to a consensus regarding implantable medication, the treatment plan could be implemented in 15 min during the same outpatient visit. This would require only one procedure semi-annually, rather than the 6 (decanoate) to 12 (microsphere) necessary for depot injections. Consequently, it is important to note that although implants would provide improved convenience and access to medication, they would not replace the need for patients to interact with psychiatrists and therapists. Rather, improved medication adherence and continuous access to medication would allow patients and their physicians to focus on issues other than medication adherence in the service of a more comprehensive treatment plan.

As the desire has increased for long-term delivery of medication to treat countless chronic illnesses, it is also beneficial to explore sustained delivery options for a variety of pharmaceuticals, especially those that are limited by the chemistry of decanoates. A need for lasting medication systems has already been expressed in several fields, including treatments for cardiovascular disease (Chui et al. 2003), diabetes (Boccuzzi et al. 2001), cancer (Natsugoe et al. 1997) and HIV/AIDS (Andrews and Friedland 2000; Melbourne et al. 1999; Tucker et al. 2003). Accordingly, the ability of surgically implantable long-term delivery systems to incorporate other medications for the treatment of psychiatric and non-psychiatric illnesses has been further explored in complimentary studies associated with our group (Siegel et al. 2006).

In summary, we advocate the use of long-term surgically implantable formulations of antipsychotic medications for improved adherence in the treatment of psychotic disorders. This study demonstrates the behavioral and pharmacokinetic feasibility of such a system allowing release for up to one year. Further in vivo and in vitro trials of implantable formulations with a variety of medications could be examined to utilize other antipsychotic agents and treat a large number of chronic disorders. 
Acknowledgements The Stanley Medical Research Institute funded this research. Intellectual property related to haloperidol implants developed at the University of Pennsylvania is owned by the University. Research by NRS and JMS supported by MH42228 and MH01436. All experiments comply with "Principles of Laboratory Animal Care" and conform to University of Pennsylvania IACUC standards.

\section{References}

Adams CE, Fenton MK, Quraishi S, David AS (2001) Systematic meta-review of depot antipsychotic drugs for people with schizophrenia. Br J Psychiatry 179:290-299

Andrews L, Friedland G (2000) Progress in HIV therapeutics and the challenges of adherence to antiretroviral therapy. Infect Dis Clin North Am 14:901-928

Ayuso-Gutierrez JL, del Rio Vega JM (1997) Factors influencing relapse in the long-term course of schizophrenia. Schizophr Res 28:199-206

Boccuzzi SJ, Wogen J, Fox J, Sung JC, Shah AB, Kim J (2001) Utilization of oral hypoglycemic agents in a drug-insured U.S. population. Diabetes Care 24:1411-1415

Chui MA, Deer M, Bennett SJ, Tu W, Oury S, Brater DC, Murray MD (2003) Association between adherence to diuretic therapy and health care utilization in patients with heart failure. Pharmacotherapy 23:326-332

Corriss DJ, Smith TE, Hull JW, Lim RW, Pratt SI, Romanelli S (1999) Interactive risk factors for treatment adherence in a chronic psychotic disorders population. Psychiatry Res 89:269-274

Curtis H (1983) Biology, Fourth edn. Worth, New York

Dash AK, Cudworth GC, 2nd (1998) Therapeutic applications of implantable drug delivery systems. J Pharmacol Toxicol Methods 40:1-12

Davis JM, Chen N, Glick ID (2003) A meta-analysis of the efficacy of second-generation antipsychotics. Arch Gen Psychiatry 60:553564

Fischel-Ghodsian F, Newton JM (1993) Analysis of drug release kinetics from degradable polymeric devices. J Drug Target 1:5157

Gastpar M, Masiak M, Latif MA, Frazzingaro S, Medori R, Lombertie ER (2005) Sustained improvement of clinical outcome with risperidone long-acting injectable in psychotic patients previously treated with olanzapine. J Psychopharmacol 19:32-38

Geddes J, Freemantle N, Harrison P, Bebbington (2000) Atypical antipsychotics in the treatment of schizophrenia: systematic overview and meta-regression analysis. BMJ 321:1371-1376

Gould TJ, Bizily SP, Tokarczyk J, Kelly MP, Siegel SJ, Kanes SJ, Abel T (2004) Sensorimotor gating deficits in transgenic mice expressing a constitutively active form of Gs alpha. Neuropsychopharmacology 29:494-501

Harris NR, Lovell K, Day JC (2002) Consent and long-term neuroleptic treatment. J Psychiatr Ment Health Nurs 9:475-482

Irani F, Dankert M, Brensinger C, Bilker WB, Nair SR, Kohler CG, Kanes SJ, Turetsky BI, Moberg PJ, Ragland JD, Gur RC, Gur RE, Siegel SJ (2004) Patient attitudes towards surgically implantable, long-term delivery of psychiatric medicine. Neuropsychopharmacology 29:960-968

Kane JM, Aguglia E, Altamura AC, Ayuso Gutierrez JL, Brunello N, Fleischhacker WW, Gaebel W, Gerlach J, Guelfi JD, Kissling W, Lapierre YD, Lindstrom E, Mendlewicz J, Racagni G, Carulla LS, Schooler NR (1998) Guidelines for depot antipsychotic treatment in schizophrenia. European Neuropsychopharmacology Consensus Conference in Siena, Italy. Eur Neuropsychopharmacol 8:55-66

Kitchell JP, Wise DL (1985) Poly(lactic/glycolic acid) biodegradable drug-polymer matrix systems. Methods Enzymol 112:436-448
Lieberman JA, Stroup TS, McEvoy JP, Swartz MS, Rosenheck RA, Perkins DO, Keefe RS, Davis SM, Davis CE, Lebowitz BD, Severe J, Hsiao JK (2005) Effectiveness of antipsychotic drugs in patients with chronic schizophrenia. N Engl J Med 353:12091223

Martin SD, Libretto SE, Pratt DJ, Brewin JS, Huq ZU, Saleh BT (2003) Clinical experience with the long-acting injectable formulation of the atypical antipsychotic, risperidone. Curr Med Res Opin 19:298-305

McCombs JS, Nichol MB, Stimmel GL, Shi J, Smith RR (1999) Use patterns for antipsychotic medications in Medicaid patients with schizophrenia. J Clin Psychiatry 60(Suppl 19):5-11; discussion $12-3$

Melbourne KM, Geletko SM, Brown SL, Willey-Lessne C, Chase S, Fisher A (1999) Medication adherence in patients with HIV infection: a comparison of two measurement methods. AIDS Read 9:329-338

Menzin J, Boulanger L, Friedman M, Mackell J, Lloyd JR (2003) Treatment adherence associated with conventional and atypical antipsychotics in a large state medicaid program. Psychiatr Serv 54:719-723

Natsugoe S, Kumanohoso T, Tokuda K, Shimada M, Mueller J, Nakamura K, Yamada K, Fukuzaki H, Aikou T (1997) Controlled release of cisplatin incorporated into biodegradable poly-D,L-lactic acid. Anticancer Res 17:1957-1960

Okada H, Toguchi H (1995) Biodegradable microspheres in drug delivery. Crit Rev Ther Drug Carr Syst 12:1-99

Olfson M, Mechanic D, Hansell S, Boyer CA, Walkup J, Weiden PJ (2000) Predicting medication noncompliance after hospital discharge among patients with schizophrenia. Psychiatr Serv 51:216-222

Regenthal R, Kunstler U, Junhold U, Preiss R (1997) Haloperidol serum concentrations and D2 dopamine receptor occupancy during low-dose treatment with haloperidol decanoate. Int Clin Psychopharmacol 12:255-261

Robinson DG, Woerner MG, Alvir JM, Bilder RM, Hinrichsen GA, Lieberman JA (2002) Predictors of medication discontinuation by patients with first-episode schizophrenia and schizoaffective disorder. Schizophr Res 57:209-219

Rosenheck R, Perlick D, Bingham S, Liu-Mares W, Collins J, Warren S, Leslie D, Allan E, Campbell EC, Caroff S, Corwin J, Davis L, Douyon R, Dunn L, Evans D, Frecska E, Grabowski J, Graeber D, Herz L, Kwon K, Lawson W, Mena F, Sheikh J, Smelson D, Smith-Gamble V (2003) Effectiveness and cost of olanzapine and haloperidol in the treatment of schizophrenia: a randomized controlled trial. Jama 290:2693-2702

Sabel BA, Dominiak P, Hauser W, During MJ, Freese A (1990) Levodopa delivery from controlled-release polymer matrix: delivery of more than 600 days in vitro and 225 days of elevated plasma levels after subcutaneous implantation in rats. J Pharmacol Exp Ther 255:914-922

Seeman MV (2001) Clinical trials in psychiatry: do results apply to practice? Can J Psychiatry 46:352-355

Sharon AC, Wise DL (1981) Development of drug delivery systems for use in treatment of narcotic addiction. NIDA Res Monogr 28:194-213

Siegel SJ, Kahn JB, Metzger K, Winey KI, Werner K, Dan N (2006) Effect of drug type on the degradation rate of PLGA matrices. Eur J Pharm Biopharm 64(3):287-293

Siegel SJ, Winey KI, Gur RE, Lenox RH, Bilker WB, Ikeda D, Gandhi N, Zhang WX (2002) Surgically implantable long-term antipsychotic delivery systems for the treatment of schizophrenia. Neuropsychopharmacology 26:817-823

Svarstad BL, Shireman TI, Sweeney JK (2001) Using drug claims data to assess the relationship of medication adherence with hospitalization and costs. Psychiatr Serv 52:805-811 
Swerdlow NR, Geyer MA (1998) Using an animal model of deficient sensorimotor gating to study the pathophysiology and new treatments of schizophrenia. Schizophr Bull 24:285-301

Talbot K, Eidem WL, Tinsley CL, Benson MA, Thompson EW, Smith RJ, Hahn CG, Siegel SJ, Trojanowski JQ, Gur RE, Blake DJ, Arnold SE (2004) Dysbindin-1 is reduced in intrinsic, glutamatergic terminals of the hippocampal formation in schizophrenia. J Clin Invest 113:1353-1363
Tucker JS, Burnam MA, Sherbourne CD, Kung FY, Gifford AL (2003) Substance use and mental health correlates of nonadherence to antiretroviral medications in a sample of patients with human immunodeficiency virus infection. Am J Med 114:573-580

Velligan DI, Lam F, Ereshefsky L, Miller AL (2003) Psychopharmacology: perspectives on medication adherence and atypical antipsychotic medications. Psychiatr Serv 54:665-667 